\title{
Culture in vitro d'ovules non fécondés et d'em- bryons prélevés 8 jours après pollinisation chez le pommier cultivé (Malus $\times$ domestica Borkh.)
}

\author{
Yong Xiang ZHANG \& Yves LESPINASSE \\ I.N.R.A., Station d'Amélioration des Espèces fruiticres et ornementales, Beatconzé, F 49000 Angers
}

\begin{abstract}
RÉSUMÉ Des ovules non fécondés et des ovules prélevés 8 jours après pollinisation chez 3 génotypes de pommier "R I-49", "Lodi » et «Erovaln » ont été mis en culture in vitro sur des milieux comportant le milieu de base de MURASHIGE \& SKOOG complétć avec diverses combinaisons en phytohormones et saccharose. Deux embryoïdes chez "R - 49 " et des cals chez "Lodi" et "Erovan » ont été induits après culture des ovules non lécondés. De nombreuses plantes issues soit de germination directe, soit de néoformation de bourgcons adventifs ont été obtenues sur les 3 génotypes par culture des embryons prélevés 8 jours après pollinisation : toutes ces plantes présentent un phénotype normal. comparable au phénotype de plantes diplö̈des. L'intérêt de la culture d’embryons prélevés à un stade très précoce est discuté.
\end{abstract}

Mots clés additionnels : Embryö̈le, cal, embryon immature, haplö̈le, dormance emhrronnaire.

\begin{abstract}
In vitro culture of unfertilized ovules and embryos collected 8 days after pollination in Malus $\times$ domestica Borkh.

Unfertilized ovules and ovules collected 8 days after pollination on three apple cultivars "R $1-49$ ". "Lodi" and "Erovan" were cultivated in vitro on media made with MURASHIGi \& SKOOG basic medium complemented with various phytohormones and sucrose combinations. Two embryoids from "RI-49" and calluses from "Lodi" and "Erovan" were induced after culture of the unfertilized ovules. Plants from either direct germination or ncoformation of adventitious shoots were obtained in the three cultivars by culture of immature embryos collected 8 days after pollination; all these plants showed. normal phenotype, similar to that of diploid plants. The value of in vitro culture of very young embryos is discussed.
\end{abstract}

Additional key words : Embrroid, callus, immature embryo, haploid, embryo dormancy.

\section{INTRODUCTION}

La gynogenèse in vitro par culture d'ovaires ou d'ovules non fécondés est l'une des voies d'haplö̈disation chez les végétaux.Depuis la $\mathrm{I}^{\text {re }}$ réussite obtenue chez l'orge en 1976 (SAN) de nombreux succès ont été enregistrés chez diverses espèces (SAN \& GELEBART, 1986). Pourtant, cette voie d'haploïdisation ne paraît pas avoir été essayée chez les arbres fruitiers. Une partie des résultats rapportés dans cette étude concerne la culture d'ovaires et d'ovules non fécondés de pommier.

La culture d'embryons matures et immatures pour l'amélioration variétale des arbres fruitiers est un outil dont l'intérêt est variable d'une espèce à l'autre (RAM-
MING, 1983): sauvetage des embryons sur des variétés de maturité très précoce comme chez les Prunus (Tukfy, 1933; Monft, 1968), ou levée de la dormance pour raccourcir le cycle d'amélioration variétale comme chez les Malus (NICKELL, 1951). KOUIDER et al. (1984 et 1985) ont tenté de cultiver in vitro de très jeunes embryons de pommier; ces auteurs ont pour cela effectué des prélèvements successifs dès le stade « 8 jours après l'anthèse ». Ils n'ont réussi à obtenir des bourgeonnements adventifs que sur des embryons prélevés 6 semaines et demie après l'anthèse. JAMES et al. (1984) n'ont pu obtenir des embryons adventifs qu'à partir d'embryons âgés de 50 jours à compter de l'anthèse. C'est pourquoi l'autre partie de notre étude concerne la culture d'embryons prélevés 8 jours après pollinisation. 


\section{MATÉRIEL ET MÉTHODE}

\section{A. Matériel végétal}

Les travaux ont été réalisés en 1986 et en 1987 sur les génotypes suivants :

1. «R 1-49» en 1986; il s'agit d'une sélection de première génération d'autofécondation de la variété "Golden Delicious".

2. Les variétés « Lodi » et «Erovan» en 1987; ces 2 variétés ne sont pas apparentés entre elles ni avec "Golden Delicious".

Dans le cas de la culture d'embryons prélevés 8 jours après pollinisation, il s'agit d'embryons issus d'autofécondation chez « R I-49 » et d'embryons issus de fécondation croisée chez «Erovan" et "Lodi»; le pollen utilisé alors provient de l'hybride «TNR31-35», homozygote dominant " $\mathrm{RR}$ » pour la couleur rouge des organes végétatifs et reproducteurs (LESPINASSE \& GODiCHEAU, 1980).

\section{B. Prélèvement et mise en culture}

Les ovules non fécondés ont été prélevés juste avant l'anthèse et ceux qui ont été fécondés, 8 jours après pollinisation. Ils ont été désinfectés suivant le processus déjà décrit (ZHANG et al., 1987).

Pour les ovules non fécondés, 3 modes de mise en culture ont été testés en 1986 :

1. Mise en culture de la totalité de l'ovaire ( 3 par boîte de Petri de $55 \mathrm{~mm}$ de diamètre).

2. Mise en culture d'ovules dégagés par excision de la paroi externe de l'ovaire mais encore attachés au tissu placentaire ( 5 ovaires excisés par boîte).

3. Mise en culture d'ovules isolés ( 20 par boîte).

Les 2 derniers modes de mise en culture ont nécessité une dissection des ovaires en condition d'aseptie sous une loupe binoculaire avec des aiguilles très fines.

En 1987, seul le mode 2 a été retenu en tenant compte des résultats de 1986 .

Tous les ovules fécondés prélevés au cours des 2 années, ont été traités suivant le $2^{\mathrm{C}}$ mode de mise en culture.

\section{Culture in vitro}

Les différents milieux utilisés sont regroupés dans le tableau 1:

Les essais réalisés en 1986 correspondent aux milieux $\mathrm{G}_{1}, \mathrm{G}_{2}$ et $\mathrm{G}_{3}$.

En 1987, les milieux $G_{4}$ à $G_{13}$ sont destinés à la culture d'ovules non fécondés; les milieux $G_{13}, G_{14}$ et $G_{15}$ sont utilisés pour la culture d'ovules fécondés. Le $\mathrm{pH}$ des 15 milieux est ajusté à $5,8 \pm 0,1$ avant leur stérilisation à l'autoclave pendant $20 \min$ à $115^{\circ} \mathrm{C}$.

Après leur mise en culture, les ovules fécondés et non fécondés ont été placés à $24^{\circ} \mathrm{C} \pm 1$ ( 1 traitement en 1987 sur ovules non fécondés a été conduit à $29^{\circ} \mathrm{C} \pm 1$ ) à l'obscurité pour les 4 premières semaines, avec une photopériode de $16 \mathrm{~h}$ par la suite.

\section{RÉSULTATS}

\section{A. Culture des ovules non fécondés}

\section{Réponse des différents modes de mise en culture}

Lors des expériences conduites en 1986, aucune réponse n'a été observée sur les ovaires cultivés, sauf quelques cals somatiques apparus au niveau de la coupe basale. Quand on a disséqué les ovaires 2 mois après la mise en culture, les ovules étaient tous de couleur noire et dégénérés. De plus, nous avons noté 2 difficultés spécifiques à la culture de la totalité de l'ovaire, d'une part, beaucoup de contaminations, d'autre part, beaucoup de substances polyphénoliques excrétées. Sur les ovaires excisés, les ovules se sont développés tout de suite après la mise en culture et ont augmenté rapidement de volume. Si les ovules ainsi développés restent sur le tissu placentaire, ils deviennent progressivement bruns, puis noirs. Par conséquent, en 1987, 2 à 3 semaines après la mise en culture, nous avons détaché du tissu placentaire les ovules gonflés et les avons transférés dans une nouvelle boîte de même milieu. Quant aux ovules isolés dès la mise en culture, ils ont soit évolué en volume, mais très lentement, soit dégénéré après noircissement.

\section{Production de cals et d'embryoüdes}

Après environ 1 mois d'incubation quel que soit le mode de traitement, les ovules n'évoluent plus en volume. Des cals se sont formés progressivement. Le tableau 2 montre les résultats obtenus en 1987. Le taux de callogenèse sur tous les milieux confondus est de 15,9 p. 100 pour « Lodi » et 1,1 p. 100 pour «Erovan». Il y a une nette différence entre les 2 génotypes au niveau de la callogenèse. Dans la plupart des cas, les cals se forment du côté mycropylaire, exactement au point de rupture de l'ovule. Tous ces cals ont une origine incertaine : ils ont été repiqués sur des milieux favorables à la régénération, mais aucune organogenèse n'a été observée jusqu'à présent.

En 1986, 2 embryoïdes ont été obtenus 3 mois après la mise en culture (fig. 1) ; ils sont issus d'ovules de « R 149 » isolés dès le départ, cultivés initialement sur le milieu $\mathrm{G}_{2}$ pendant 2 mois et demi puis repiqués sur un milieu composé de $\mathrm{MS}+\mathrm{BAP}, 8,9 \mu \mathrm{M}+\mathrm{GA}_{3}$, $0,6 \mu \mathrm{M}+$ acide indolylbutyrique (AIB), $2,5 \mu \mathrm{M}$. Ces 2 embryoïdes n'ont malheureusement pas évolué depuis.

\section{Influence des milieux et des conditions de culture}

La température élevée $\left(29^{\circ} \mathrm{C} \pm 1\right)$ semble à la fois bénéfique au développement des ovules et défavorable à la production de cals sur le tissu placentaire par rapport à la température employée d'ordinaire $\left(24^{\circ} \mathrm{C} \pm 1\right)$; ceci est vrai au moins pour la première période d'incubation des ovules. Les concentrations élevées (175,4 à $350,9 \mathrm{mM}$ ) de saccharose peuvent limiter la production de cals somatiques. En effet, beaucoup de cals se sont formés sur le tissu placentaire et sur le milieu $G_{11}$ contenant $87,6 \mathrm{mM}$ de saccharose chez les deux génotypes testés en 1987 ; cela empêche le développement des 
TABLEAU 1

Milieux de culture étudiés en 1986 of 1987.

Culture media tested in 1986 and 1987.

\begin{tabular}{|c|c|c|c|c|c|c|c|c|c|c|c|c|c|c|c|c|}
\hline \multirow{2}{*}{\multicolumn{2}{|c|}{ Composition (1) }} & \multicolumn{15}{|c|}{ Milieux de culture } \\
\hline & & $\mathrm{G}_{1}$ & $\mathrm{G}_{2}$ & $\mathrm{G}_{3}$ & $\mathrm{G}_{4}$ & $\mathrm{G}_{5}$ & $\mathrm{G}_{6}$ & $\mathrm{G}_{7}$ & $\mathrm{G}_{8}$ & $\mathrm{G}_{y}$ & $\mathrm{G}_{10}$ & $\mathrm{G}_{11}$ & $\mathrm{G}_{12}$ & $\mathrm{G}_{1,3}$ & $\mathrm{G}_{14}$ & $\mathrm{G}_{15}$ \\
\hline \multirow{2}{*}{ MS } & macro-éléments & 1 & $1 / 2$ & 1 & 1 & 1 & 1 & 1 & 1 & 1 & 1 & 1 & 1 & $1 / 2$ & 1 & $1 / 2$ \\
\hline & micro-ćléments & 1 & 1 & 1 & 1 & 1 & 1 & 1 & 1 & 1 & 1 & 1 & 1 & $1 / 2$ & 1 & $1 / 2$ \\
\hline Vitamines & & MS & NN & MS & MS & MS & MS & MS & MS & MS & MS & MS & MS & $\mathrm{P}(2)$ & MS & MS \\
\hline $\begin{array}{l}\text { Composants organiques } \\
\text { de KouIDER (3) }\end{array}$ & & 1 & & & & & & & & & & & & & & \\
\hline $\begin{array}{l}\text { Hydrolysat de caséine } \\
\left(\mathrm{mg} \mathrm{1^{-1 } )}\right.\end{array}$ & & 500 & & & & & & & & & & & & & & \\
\hline Agar-agar $(\%)$ & & 0,7 & 0,7 & 0,7 & 0,7 & 0,7 & 0,7 & 0,7 & 0,7 & 0.7 & 0,7 & 0,7 & 0,7 & 0,7 & 0,7 & 0,7 \\
\hline Saccharose $(\mathrm{mM})$ & & 292 & 292 & 292 & 175,4 & 175,4 & 175,4 & 175,4 & 175,4 & 175,4 & 175,4 & 87,6 & 350,8 & 175,4 & 87,6 & 87,6 \\
\hline $2,4-\mathrm{D}(\mu \mathrm{M})$ & & 4,5 & 4,5 & 1,8 & 4,5 & & & & & & & & & & & \\
\hline $\mathrm{AIA}(\mu \mathrm{M})$ & & & & 11,4 & & 5,7 & & & & & & & & & & \\
\hline ANA $(\mu \mathrm{M})$ & & & & & & & 5,4 & 5,4 & 0,5 & 5,4 & 5,4 & 5,4 & 5,4 & 5,4 & & 2,7 \\
\hline $\mathrm{BAP}(\mu \mathrm{M})$ & & 17,4 & 8,9 & & 8,9 & 8,9 & & 8,9 & 8,9 & 2,2 & 22,2 & 8,9 & 8,9 & 8,9 & & 4,4 \\
\hline
\end{tabular}

Kinétine $(\mu \mathrm{M})$

0,9

9,3

Sulfate d'adénine

$(\mu \mathrm{M})$

108,6

$\mathrm{GA}_{3}(\mu \mathrm{M})$

(1) Liste des abréviations: MS (Murashige \& Skoog, 1962); NN (Nitsch J.P. \& Nitsch C., 1969); 2,4-D (Acide, 2,4dichlorophénoxyacétique); AIA (Acide indole-3-acétique); ANA (Acide $\alpha$-naphthylacétique); BAP (benzylaminopurine); GA 3 (Acide gibberellique).

(2) Vitamines « $\mathrm{P} »$ : en $\mathrm{mg} 1{ }^{1}$, myoinositol, 100 ; glycine, 4 ; chlorure de choline, 0,4 ; acide folique, 0,4 ; riboflavine, 0.4 ; biotine, 0,04 ; thiamine, 30 ; acide nicotinique, 10 ; pyridoxine, 1,0 .

(3) Composants organiques de Kouider (Kouider et al., 1984) : $\mathrm{cn} \mathrm{mg} \mathrm{l}^{-1}$, acide ascorbique, 20 ; P-amino acide benzoïque, 1 ; chlorure de choline, 0,5 ; pantothenate de Ca, 0,5 ; extrait de malt, 400 ; lait de coco, $40 \mathrm{ml} \mathrm{l}$ ' .

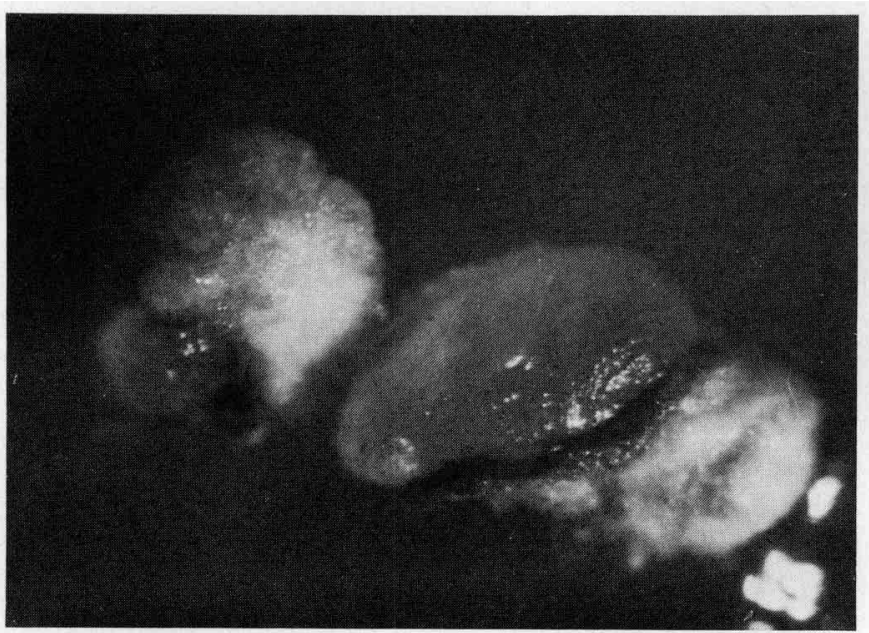

Figure 1

Embryoïdes obtenus 3 mois après la mise en culture d'ovules non fécondés chez "RI-49".

Embryoids obtained 3 months after culture of unfertilized ovules in "Rl-49".

ovules. Quant aux concentrations des phytohormones, nous sommes encore loin de les maîtriser, mais nous pouvons quand même déceler des tendances. Pour la callogenèse chez « Lodi » comme chez « Erovan », l'influence des auxines est plus marquée et régulière que celle des cytokinines : $4,5 \mu \mathrm{M}$ de $2,4-\mathrm{D}\left(\mathrm{G}_{4}\right)$ et $5,4 \mu \mathrm{M}$ d'ANA $\left(G_{7}\right)$ produisent davantage de cals que $5,7 \mu \mathrm{M}$ d'AIA $\left(G_{5}\right)$; de même $5,4 \mu \mathrm{M}$ d'ANA $\left(G_{7}\right)$ est plus favorable que $0,5 \mu \mathrm{M}$ d'ANA $\left(\mathrm{G}_{8}\right)$ (tabl. 2).

\section{B. Culture d'ovules fécondés (prélevés 8 jours après pollinisation)}

\section{Expériences conduites en 1986}

Cent cinquante ovules ont été cultivés sur chacun des 3 milieux testés. Au total, 6 embryons dont 2 sur $G_{1}, 1$ sur $G_{2}$ et 3 sur $G_{3}$ (tabl. 3 ) ont émergé de l'ovule 3 mois environ après la mise en culture sans repiquage. Parmi ceux-ci, 5 chlorophylliens ont germé aussitôt et 1 de couleur blanche est resté bloqué. Les 5 plantes en croissance se développent très bien sur un milieu composé de $\mathrm{MS}+\mathrm{BAP}, \quad 4,4 \mu \mathrm{M}+\mathrm{AIB}$, $0,5 \mu \mathrm{M}+\mathrm{GA}_{3}, \quad 1,5 \mu \mathrm{M}$. Après leur enracinement (fig. 2) sur un milieu de base (1/2 MS macro- et micro- 
TABLEAU 2

Culture d'ovules non fécondés; taux de callogenèse obtenu en 1987.

Culture of unfertilized ovules; rate of callus formation obtained in 1987.

\begin{tabular}{|c|c|c|c|c|c|c|}
\hline \multirow{3}{*}{ Milieu } & \multicolumn{2}{|c|}{ LODI } & \multicolumn{4}{|c|}{ EROVAN } \\
\hline & & d'ovules & \multirow{2}{*}{$\begin{array}{c}\text { Taux } \\
\text { de callogenèse }\end{array}$} & \multicolumn{2}{|c|}{ Nombre d'ovules } & \multirow[b]{2}{*}{ Taux de callogenèse } \\
\hline & $\begin{array}{l}\text { mis } \\
\text { en culture }\end{array}$ & $\begin{array}{l}\text { avec formation } \\
\text { de cals }\end{array}$ & & $\begin{array}{c}\text { mis } \\
\text { en culture }\end{array}$ & $\begin{array}{l}\text { avec formation } \\
\text { de cals }\end{array}$ & \\
\hline $\mathrm{G}_{4}$ & 300 & 96 & 32 & 300 & 8 & 2,7 \\
\hline $\mathrm{G}_{5}$ & 300 & 2 & 0,7 & 300 & 1 & 0,3 \\
\hline $\mathrm{G}_{6}$ & 300 & 65 & 21,7 & 300 & 3 & 1,0 \\
\hline $\mathrm{G}_{7}$ & 300 & 82 & 27,2 & 300 & 7 & 2,3 \\
\hline $\mathrm{G}_{8}$ & 300 & 19 & 6,3 & 300 & 1 & 0,3 \\
\hline $\mathrm{G}_{9}$ & 300 & 58 & 19,3 & 300 & 8 & 2,7 \\
\hline $\mathrm{G}_{10}$ & 300 & 79 & 26,3 & 300 & 0 & 0 \\
\hline $\mathrm{G}_{11}$ & 300 & 28 & 9,3 & 300 & 1 & 0,3 \\
\hline $\mathrm{G}_{12}$ & 300 & 67 & 22,3 & 300 & 0 & 0 \\
\hline $\mathrm{G}_{13}$ & 300 & 0 & 0 & 300 & 1 & 0,3 \\
\hline$* \mathrm{G}_{7}$ & 300 & 30 & 10 & 300 & 6 & 2,0 \\
\hline Total & 3300 & 526 & 15,9 & 3300 & 36 & 1,1 \\
\hline
\end{tabular}

* expérience conduite à $29^{\circ} \mathrm{C} \pm 1$.

éléments + thiamine, $0,4 \mathrm{mg} \mathrm{l}^{-1}$ ) complété soit avec $0,5 \mu \mathrm{M}$ d'AIB, soit avec 2,9 à $5,7 \mu \mathrm{M}$ d'AIA, ils ont été transférés en serre après 4 semaines d'acclimatation; leur reprise est bonne. Toutes ces plantes issues d'autofécondation de «R1-49» présentent un phénotype normal, comparable au phénotype des plantes diploïdes.

\section{Expériences conduites en 1987}

Le tableau 3 montre la mise en culture effectuée et les résultats obtenus. 22 embryons ont été observés sur
«Lodi » et 9 sur «Erovan ». Il faut souligner que ces 31 embryons sont de couleur blanche. De plus, ils semblent anormaux : certains possèdent de nombreux cotylédons, d'autres ont des cotylédons déformés. En conséquence, seulement 2 sur les 31 ont germé directement et donné des plantes. Les embryons qui n'ont pas pu germer ont été transférés sur des milieux favorables à la néoformation. Après 1 à 2 mois de culture, la néoformation a eu lieu sur 9 embryons. Le nombre de bourgeons néoformés par embryon est toujours très important; ils forment un bouquet sur lequel on peut difficilement les dénombrer. Tous ces bourgeons néoformés sur «Lodi » et "Erovan» sont de couleur rouge ; ils sont donc hybrides et diploïdes.

TABLEAU 3

Culture d'ovules fécondés (prélevés 8 jours après pollinisation); nombre d'embryons obtenus en 1986 et 1987. Culture of fertilized ovules (collected 8 davs after pollination); number of embryos obtained in 1986 and 1987.

\begin{tabular}{|c|c|c|c|c|c|}
\hline \multirow{2}{*}{ Génotype } & \multirow{2}{*}{ Milieu } & \multirow{2}{*}{$\begin{array}{l}\text { Nombre d'ovules } \\
\text { mis en culture }\end{array}$} & \multicolumn{3}{|c|}{$\begin{array}{c}\text { Nombre d'embryons } \\
\text { obtenus }\end{array}$} \\
\hline & & & total & $\begin{array}{l}\text { ayant germé } \\
\text { dircctement }\end{array}$ & $\begin{array}{c}\text { avec bourgeons } \\
\text { adventifs }\end{array}$ \\
\hline \multirow{3}{*}{ R I-49 } & $\mathrm{G}_{1}$ & 150 & 2 & 1 & - \\
\hline & $\mathrm{G}_{2}$ & 150 & 1 & 1 & - \\
\hline & $\mathrm{G}_{3}$ & 150 & 3 & 3 & - \\
\hline \multirow{3}{*}{ LODI } & $\mathrm{G}_{13}$ & 150 & 20 & 2 & 6 \\
\hline & $\mathrm{G}_{14}$ & 150 & I & - & 1 \\
\hline & $G_{15}$ & 150 & 1 & - & - \\
\hline \multirow{3}{*}{ EROVAN } & $\mathrm{G}_{13}$ & 150 & 6 & - & 1 \\
\hline & $G_{14}$ & 150 & 1 & - & 1 \\
\hline & $\mathrm{G}_{15}$ & 150 & 2 & - & - \\
\hline
\end{tabular}




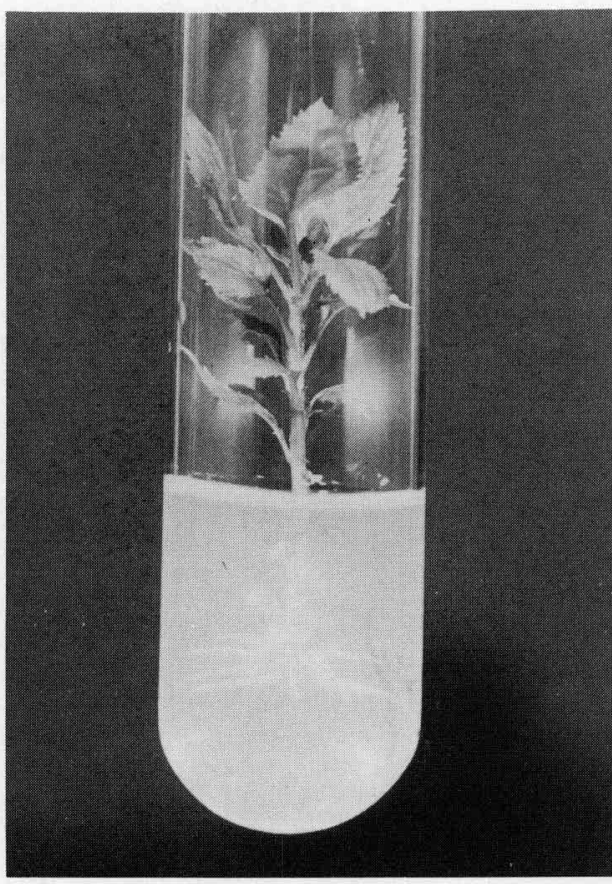

Figure 2

Plante enracinée issue de la culture d'un ovule prélevé 8 jours après pollinisation chez «R $R 1-49$ ».

Rooted plant from the culture of an ovule collected 8 days after pollination in " $R I-49$ ".

\section{DISCUSSION ET CONCLUSION}

\section{A. La culture d'ovules non fécondés}

La culture d'ovules non fécondés et l'obtention de plantes gynogénétiques in vitro n'a jamais été réalisée chez les arbres fruitiers; la présente étude constitue donc une première tentative. Notre travail nous a permis de mettre en évidence un mode adéquat de mise en culture; c'est un premier pas nécessaire. Nos résultats ont montré en outre que la mise en culture d'ovaires dans leur totalité pratiquée couramment chez les graminées (YANG \& ZHOU, 1982) ne convient vraisemblablement pas au pommier ; la mise en culture d'ovules isolés dès le départ est réalisable mais délicate en pratique en raison de la petite taille des ovules. Nous avons ainsi adopté seulement la mise en culture des ovules excisés c'est-à-dire des ovules dégagés mais solidaires du tissu placentaire; la séparation a lieu 2 à 3 semaines plus tard. Les résultats obtenus ont fourni les premières indications concernant l'influence des températures, de la teneur en saccharose et en phytohormones et des génotypes. L'obtention de 2 embryoïdes chez « R 1-49» et d'un faible taux de callogenèse chez "Erovan» et "Lodi » n'est qu'une première étape; beaucoup d'efforts sont encore nécessaires pour la réussite de la gynogenèse in vitro.

\section{B. Les ovules prélevés 8 jours après pollinisation}

Cinq des 6 embryons obtenus en 1986 étaient chlorophylliens dès leur apparition; ces embryons ont pu germer et croître très rapidement. Toutefois en 1987, nous n'avons obtenu que des embryons de couleur blanche; seuls 2 d'entre eux ont germé directement. Cette très nette différence au niveau de la qualité des embryons obtenus et du pouvoir germinatif reste difficile à expliquer; elle pourrait être attribuée aux génotypes et (ou) à l'influence des milieux. Il convient de souligner que l'une des différences quant aux milieux utilisés lors des 2 années d'expérimentation réside dans la quantité de saccharose : $292 \mathrm{mM}$ pour les milieux de 1986 et $87,6 \mathrm{mM}$ pour ceux de 1987 . On peut imaginer que la pression osmotique est l'un des facteurs à considérer.

Le résultat original de ces 2 années d'études est l'obtention de plantes par culture d'ovules prélevés 8 jours après pollinisation. Ceci n'a jamais été rapporté chez le pommier et peut présenter plusieurs avantages en amélioration variétale ainsi que pour des études physiologiques et embryologiques. Quant à l'application dans les programmes d'amélioration variétale, on peut envisager la culture d'embryons immatures à un stade très précoce, par exemple, dans le cadre du programme d'hybridation pour la résistance au feu bactérien. Après l'obtention de plantes et la multiplication in vitro, l'inoculation par l'agent pathogène et la sélection précoce pourraient être effectuées au cours de la même année; les individus sélectionnés seraient alors micropropagés rapidement. Tout cela permettrait de raccourcir le cycle d'amélioration variétale et de simplifier les manipulations: absence de stratification, semis en serre puis confinement pour l'inoculation. La culture d'ovules prélevés 1 semaine après pollinisation peut être une technique appréciable pour les études conduites en physiologie de la graine. Le mécanisme de la dormance chez le pommier est encore mal connu; toutefois, il est admis que la dormance s'installe, au cours du développement des embryons, à un stade très précoce (THEVENOT \& DURAND, 1973 ; THEVENOT \& COME, 1983). La majorité des embryons obtenus en 1986 peuvent germer aussitôt; ceci signifie que la dormance chez ces embryons issus d'autofécondation ne s'est pas encore installée. D'autre part, selon THEVENot \& Durand (1973), l'état d'immaturité des embryons joue aussi un rôle dans leur germination. La possibilité de germination à un stade très précoce doit permettre donc d'aborder sous un angle nouveau ces différents phénomènes, état d'immaturité de l'embryon, installation et intensité de la dormance, ainsi que leur interaction.

Reçule 29 mars 1988 Accepté le 27 aoùt 1988. 


\section{RÉFÉRENCES BIBLIOGRAPHIQUES}

James D. J., Passey A. J., Deeming D. C., 1984. Adventitious embryogenesis and the in vitro culture of apple seed parts. J. Plant Physiol., 115, 217-229.

Kouider M., Skirvin R. M., Korban S. S., Widholm J. M., 1984 Adventitious shoot formation from Red Delicious apple cotyledons in vitro. J. Hortic. Sci.. 59 (3), 295-302.

Kouider M., Korban S. S., Skirvin R. M., Joung M., 1985. The relationship of apple embryos and their cotyledons to maturity, dormancy and the potential to form shoots in vitro. J. Amer. Soc Hortic. Sci., 110 (1), 93-96.

Lespinasse Y., Godicheau M., 1980. Création et description d'une plante haplö̈de de pommier (Malus pumila Mill.). Am. Amélior. Plantes, 30 (1), 39-44.

Monet R., 1968. Mćthode permettant l'obtention de plantes viatles à partir d'embryons de variétés très précoces chez le pêcher. Ann. Amélior. Plantes, 18 (1), 85-91.

Murashige T., Skoog F., 1962. A revised medium for rapid growth and bio-assays with tobacco tissue culture. Physiol. Plamt., 15, 47.3-497.

Nickell L. G., 1951. Embryo culture of weeping crabapple. Proc. Am. Soc. Hortic. Sci., 57, 401-405.

Nitsch J. P., Nitsch C., 1969. Haploid plants from pollen grains. Science, 163,87
Ramming D., 1983. Embryo culture. In J. N. Moore \& J. Janick, eds. Methods in fruit breeding. Purdue University Press, West Lafayette. Indiana, 136-144.

San L. H., 1976. Haplö̈des d'Hordeum vulgare L. par culture in vitro d'ovaires non fécondés. Ann. Amélior. Plantes, 26 (4), 751-754.

San L. H., Gelebart P., 1986. Production of gynogenetic haploids, 305-322. ch. 15. In I. K. Basil, ed. Cell culture and somatic cell genetics of plants, Vol. 3, Academic Press.

Thévenot C., Durand M., 1973. Mise en évidence d'une dormance chez les embryons de pommier immatures. C. R. Acad. Agric. Fr., 908-918

Thévenot C.. Côme D., 1983. Corrélations physiologiques entre l'axe et les cotylédons des embryons dormants. Bull. Soc. Bot. Fr., 130, Actual. bot. (3-4), 79-88.

Tukey H. B., 1933. Artificial culture of sweet cherry embryos. $J$ Hered., 24, 7-12

Yang H. Y., Zhou C., 1982. In vitro induction of haploid plants from unpollinated ovaries and ovules. Theor. Appl. Genet., 63, 97-104.

Zhang Y. X., Boccon-Gibod J., Lespinasse Y., 1987. Obtention d'embryons de pommier (Malus $\times$ domestica Borkh.) après culture d’anthères. C. R. Acad. Sci., Paris, t, 305. Série III, 443-448. 